\title{
Législation des essais cliniques : préparer la révision de la Directive Européenne prévue en 2011
}

\author{
Pascal Bilbault ${ }^{1}$, Chantal Belorgey ${ }^{2}$, Faiez Zannad ${ }^{3}$, Danielle Golinelli ${ }^{4}$, Yannick Pletan ${ }^{5}$ et les participants à la \\ Table Ronde $n^{\circ} 4$ de Giens XXV ${ }^{\star}$ \\ 1 Laboratoire Boehringer Ingelheim, Reims, France \\ 2 Afssaps, Saint-Denis, France \\ 3 Centre d'Investigation Clinique, Hôpital Jeanne d'Arc, Dommartin les Toul, France \\ 4 Direction Générale de la Santé, Paris, France \\ 5 Laboratoire Pfizer, Paris, France
}

Texte reçu le 29 mars 2010 ; accepté le 23 avril 2010

\section{Mots clés :}

essais cliniques ;

directive européenne ;

recherche biomédicale ; révision ;

risque
Résumé - L'objet de cette Table Ronde était de proposer des recommandations en vue d'une prochaine révision de la Directive communautaire portant sur les essais cliniques de médicaments (2001/20/CE).

Tout en reconnaissant l'importance de faire respecter l'application de la Directive, application qui apparaît insuffisante dans certains États-membres de l'Union Européenne, il serait utile de la simplifier davantage et d'harmoniser son application dans tous les pays.

Sans recourir nécessairement à une révision, certaines dispositions de la Directive pourraient être améliorées : meilleure définition des «médicaments expérimentaux » et de ce qui doit être considéré comme «modifications substantielles » ou amélioration du fonctionnement des Comités d'Éthique (CE) au niveau de la Commission Européenne en s'appuyant davantage sur l'European Network of Research Ethics Committees (EUREC), réseau des CE existant déjà dans plusieurs pays européens.

D'autres points de la Directive nécessiteraient une révision, notamment ceux portant sur :

- La définition des rôles respectifs des CE et des Autorités Compétentes.

- La simplification de l'information de pharmacovigilance à relayer aux CE (leur donner accès à la base de données Eudravigilance sur les effets indésirables survenant en cours d'essai et ne leur envoyer que les faits nouveaux ou un résumé du Rapport Annuel de Sécurité). - La possibilité d'une autorisation communautaire de l'essai, centralisée et/ou décentralisée lorsque l'essai est multinational.

Les changements recommandés pourraient se faire dans le cadre d'un Règlement Européen, ce qui permettrait d'éviter le besoin d'une transposition ultérieure dans la règlementation de chaque pays, source de dysharmonies potentielles.

Plus spécifiquement pour des essais de promotion institutionnelle de type «stratégie thérapeutique médicament », la modulation des contraintes en fonction du «risque ajouté par la recherche » permettrait de simplifier l'organisation de l'essai concernant notamment le monitoring, la gestion des effets indésirables et l'étiquetage des médicaments.

\section{Introduction}

Depuis sa mise en application, la Directive Européenne 2001/20/CE relative aux essais cliniques sur le médicament a permis une harmonisation règlementaire entre les différents pays de l'Union Européenne, en particulier pour l'application des bonnes pratiques cliniques, la protection des personnes (notamment les patients vulnérables), la définition des conditions de réalisation des recherches (régime d'autorisation préalable, qualité des médicaments expérimentaux utilisés) et le partage

\footnotetext{
^ Pour la liste des participants, voir en fin d'article.
} 
Tableau I. Avis des investigateurs européens auteurs d'essais sur les CE les ayant évalués.

\begin{tabular}{ll}
\hline 5 questions posées aux investigateurs sur leur interaction avec les CE & Score moyen * \\
\hline - Le CE a fourni un bon soutien & $-2,5$ \\
- L'effort nécessaire pour obtenir l'autorisation nous a fait faire des progrès en matière d'éthique & $-3,7$ \\
- L'effort nécessaire pour obtenir l'autorisation nous a fait faire des progrès scientifiques & $-4,1$ \\
- L'effort nécessaire pour obtenir l'autorisation était justifié & $-1,1$ \\
- L'autorisation a été donnée dans un environnement courtois & 2,0 \\
\hline
\end{tabular}

- score entre -10 (extrêmement insatisfait) et +10 (extrêmement satisfait)

CE : Comités d'Éthique

des informations entre Autorités Compétentes (AC) des Étatsmembres (base de données EudraCT).

Cette importante avancée s'est toutefois accompagnée pour les promoteurs d'essais cliniques, institutionnels ou industriels, de lourdeurs administratives et d'une augmentation significative de la charge de travail ainsi que de divergences d'interprétation entre les États-membres.

Il était donc important à l'occasion des débats sur la nécessité d'une révision de la Directive Européenne prévue en 2011 de proposer des recommandations au niveau français.

Nous avons considéré spécifiquement le point de vue des institutionnels et des industriels ainsi que celui des Comités de Protection des Personnes (CPP) et nous avons mené la réflexion dans 3 directions :

1. Comment mieux faire appliquer la Directive actuelle dans certains pays européens?

2. Comment la simplifier davantage?

3. Comment harmoniser son application dans les différents pays?

Un chapitre est d'autre part consacré à une proposition de nouvelle approche basée sur le risque ajouté par la recherche.

Une synthèse des recommandations souhaitables au niveau européen est enfin proposée.

\section{Le point de vue des promoteurs institutionnels}

Une étude ${ }^{[1]}$ a montré le ressenti d'investigateurs européens ayant publié des essais cliniques dont ils étaient auteurs dans de grands journaux médicaux vis-à-vis des Comités d'Éthique (CE) européens qui avaient émis un avis sur ces études (tableau I). Tant sur le fond (expertise et compétence des Comités) que sur la forme (procédures de soumission et qualité des contacts) les investigateurs étaient plutôt insatisfaits.

Au plan européen, plusieurs initiatives ont été prises par les promoteurs institutionnels depuis quelques années à l'instigation notamment de réseaux d'investigateurs comme le réseau European Clinical Research Infrastructures Network (ECRIN) ou par le biais du projet Impact on Clinical Research of European Legislation (ICREL) ${ }^{[2]}$ ou du regroupement d'organismes de recherche tels que 1'European Science Foundation (ESF).

On peut souligner l'enquête d'ECRIN ${ }^{[3]}$ qui a confirmé la très grande hétérogénéité des règlementations nationales (surtout pour ce qui concerne les recherches hors médicaments) et celle de ICREL qui confirme que le nombre total d'essais sur le médicament n'a pas baissé après la mise en place de la Directive mais que les délais de mise en place se sont allongés de $24 \%$ en 3 ans avec une surcharge de travail notable. ${ }^{[2]}$

Ces initiatives concluent toutes à la nécessité de simplifier les procédures d'évaluation des essais de médicaments en Europe : souhait d'une évaluation par une AC unique, que pourrait préfigurer pour les essais cliniques de médicaments la Procédure Volontaire d'Harmonisation (PVH) mise en place à l'initiative du Clinical Trial Facilitation Group (CTFG), souhait de disposer de systèmes d'assurance harmonisés, souhait enfin de s'accorder sur une typologie des recherches basée sur le niveau de risque lié à la recherche. Le fonctionnement encore imparfait des CE, leur manque d'harmonisation en Europe et le souhait de l'avis d'un seul CE certifié par pays pour chaque protocole est aussi des sujets récurrents.

La Directive Européenne de 2001 prévoyait pourtant l'examen du protocole par un CE unique par pays. Ce point n'est pas encore appliqué dans tous les pays, notamment ceux qui sont organisés en régions (Allemagne, Espagne). Il n'a pas été démontré que la multiplicité d'avis améliorait les aspects éthique et scientifique du projet.

Au niveau français il semble devenu également plus difficile d'organiser les essais cliniques tant pour les promoteurs institutionnels que pour les promoteurs industriels depuis la mise en application de la Directive Européenne en France et cela malgré les informations disponibles au plan règlementaire.

La proposition de Loi Jardé ${ }^{[4]}$ devrait faciliter une partie des essais institutionnels par la création, pour les essais hors médicaments, d'une catégorie supplémentaire de recherches interventionnelles à risque négligeable par rapport à l'utilisation normale (élargissant la notion existante de recherches visant à évaluer les soins courants). Concernant le médicament, les études de stratégie 
thérapeutique de phase IV intéressant nombre de réseaux académiques restent toutefois des recherches biomédicales à part entière sujettes à la Directive Européenne, du fait que cette dernière ne prévoit qu'une seule catégorie de recherches interventionnelles. Ce cadre n'est pas forcément adapté en raison des contraintes administratives et règlementaires consommatrices de ressources importantes, et à l'avenir les institutionnels risquent de se détourner de ce type d'études. Une nouvelle approche basée sur le risque ajouté par la recherche pourrait permettre de diminuer le niveau de contraintes de l'essai en termes de procédures d'évaluation, de monitoring et de gestion des effets indésirables.

\section{Le point de vue des Comités de Protection des Personnes}

La place des CE est variable d'un pays européen à l'autre. Si en France les rôles respectifs du CPP et de l'AC sont clairement définis par la législation, il n'en est pas de même partout en Europe.

Une étude du CTFG a été conduite en 2008 sur les rôles et responsabilités respectives de l'AC et du CE dans la plupart des pays européens. ${ }^{[5]}$ L'information des sujets et le document de recueil du consentement sont analysés par les deux structures dans la moitié des pays concernés. Le dossier du médicament est évalué essentiellement par l'AC mais 4 pays confient cette tâche également au CE pour une partie du dossier. L'évaluation de la méthodologie est confiée aux CE dans 4 pays, à l'AC dans 3 et partagée entre les CE et l'AC dans les autres pays. La soumission est faite en parallèle aux $\mathrm{CE}$ et à l' $\mathrm{AC}$ dans la majorité des pays mais les modalités des avis vont de l'avis unique d'un CE pour tous les centres comme en France, au Portugal ou en Grèce, à des systèmes plus complexes. On peut ainsi trouver une procédure différente selon que l'étude est monocentrique avec un avis du CE local ou multicentrique avec un avis du CE central. C'est le cas par exemple en Autriche ou en Finlande. La situation de pays comme l'Allemagne ou l'Italie est complexe avec à la fois un avis de CE local et un avis de CE central. Certains aspects du dossier sont traités par une des instances, et les autres par la deuxième instance.

Au total, bien que la Directive requière un avis unique, les procédures d'émission de cet avis sont donc parfois complexes et peuvent faire appel à une ou plusieurs structures selon les pays.

Qu'en est-il des échanges entre les deux structures aussi bien pendant l'analyse du dossier que pour la transmission de l'avis d'une structure à l'autre?

Une enquête récente sur ce sujet a été réalisée par un des membres de la table ronde auprès de ses correspondants nationaux et 19 pays ont répondu.
Dans la majorité des cas il n'y a pas d'échanges entre les CE et l'AC pendant l'analyse du dossier. En revanche, la majorité des $\mathrm{CE}$ transmet l'avis émis à l'AC, la Pologne faisant exception à cette règle. Dans le sens $\mathrm{AC}$ vers $\mathrm{CE}$, les commentaires de l'AC sont automatiquement transmis en France au CPP concerné par le dossier. Les AC des autres pays ne transmettent pas leurs remarques au CE. De même, l'avis définitif de l'AC n'est transmis au CE que dans 4 pays sur 20.

Un cas particulier est représenté par l'Espagne qui, grâce à un site internet, permet des échanges permanents entre $\mathrm{CE}$ et $\mathrm{AC}$, aussi bien pendant la procédure d'examen du dossier que pour l'émission de l'avis définitif.

La France se distingue par la transmission par chaque CPP des avis négatifs à tous les autres CPP. En cas d'avis négatif du $\mathrm{CE}$, certains pays dont la France, ont prévu une procédure de second examen par un autre CE.

Un autre sujet d'amélioration possible est celui de la composition des CE. La Directive prévoit dans la composition une parité société civile et expertise scientifique qui n'est pas appliquée dans tous les pays. Le nombre de membres comme les modalités de nomination et les qualifications requises sont laissés au libre choix de chaque pays. Selon les pays les Comités peuvent comporter de 7 à 25 membres.

\section{1. Les recommandations des CPP}

Au terme de cette analyse, il semble qu'une des principales recommandations en vue d'une meilleure harmonisation serait l'examen par un seul CE par pays auprès duquel serait déposé un dossier identique pour tous les pays.

Il parait difficile d'envisager un avis éthique unique européen pour un dossier. On peut certes concevoir une approche basée sur les principes généraux du caractère éthique de la recherche par rapport à la connaissance scientifique dans le domaine concerné, mais il est indispensable de maintenir un avis éthique par pays, car cette approche est la seule qui permette de tenir compte des spécificités culturelles de chacun et de leur expression dans l'avis rendu sur les documents d'information et de consentement.

Un meilleur fonctionnement et une meilleure harmonisation entre les CE devraient pouvoir aussi être obtenus comme suit :

- Intra pays : dossier harmonisé, format unique, guichet unique, avis type, référentiels.

- Inter pays : dossier harmonisé, format unique, meilleure harmonisation de la composition des $\mathrm{CE}$ et des échanges entre les CE et AC.

- Outil électronique (communication inter $\mathrm{CE}$, accès à EudraCT).

- Formation et certification. 
Une autre recommandation serait de s'appuyer davantage sur l'European Network of Research Ethics Committees (EUREC) ${ }^{[6]}$ qui est un réseau des $\mathrm{CE}$ regroupant déjà une dizaine de pays européens en vue d'améliorer l'harmonisation des pratiques et des avis.

\section{Le point de vue des promoteurs industriels}

Le constat fait au niveau industriel rejoint les constats précédents : la Directive a été indiscutablement d'un grand apport en Europe et les laboratoires se sont organisés en conséquence pour la mise en place et la gestion de leurs essais cliniques. Dans le domaine de la pharmacovigilance, la Directive a permis de parler globalement le même langage et d'offrir, en théorie, une meilleure protection des patients en échangeant plus d'informations liées aux données de sécurité entre les parties prenantes.

La Directive a toutefois perdu en harmonisation après sa transposition dans les différents États-membres qui a introduit des différences dans son champ d'application, dans les délais d'autorisation ( « clock-stop » pendant le délai d'évaluation dans certains pays), dans les demandes de documentations complémentaires, dans les définitions du médicament expérimental et des modifications substantielles, enfin dans les déclarations d'effets indésirables introduisant de nombreuses spécificités nationales. Une enquête au niveau des maisons mères ou sièges Europe d'entreprises pharmaceutiques a confirmé les difficultés liées aux interprétations différentes selon les pays, aux avis divergents entre AC ou entre $\mathrm{AC}$ et $\mathrm{CE}$ et à l'hétérogénéité des $\mathrm{CE}$ conduisant des Comités locaux à des demandes plus scientifiques que celles des Comités nationaux par exemple.

D'une façon générale, la charge de travail s'est accentuée aussi bien pour les promoteurs que pour les AC et CE. Cette surcharge est liée aux requis réglementaires supplémentaires en rapport avec la mise en place de la Directive mais aussi à la nécessité pour les promoteurs de respecter toutes les spécificités nationales. En parallèle, cette augmentation d'activité a entraîné des coûts additionnels, en particulier associés au besoin de ressources supplémentaires.

Les CE rencontrent des difficultés de fonctionnement avec une lourdeur de plus en plus importante dans la gestion manuelle de l'ensemble des documents papier. Ces difficultés concernent particulièrement les données de vigilance avec des contraintes réelles pour revoir et analyser les documents soumis, particulièrement toutes les observations individuelles. Ces suspicions d'effets indésirables graves inattendus (SUSARs) sont transmises par les promoteurs sans aucune analyse et sont envoyées en même temps aux AC. En outre cette quantité d'informations «brutes » ne per- met pas de mettre en avant les éléments les plus pertinents, ce qui serait plus approprié en matière de protection des sujets de l'essai.

Les CE reçoivent aussi des listings d'effets indésirables graves, envoyés généralement sans commentaires et parfois sur un rythme hétérogène d'un pays à un autre (le plus souvent semestriels mais parfois trimestriels).

Quant aux Rapports Annuels de Sécurité (RAS), documents de plus en plus volumineux et qui ne requièrent aucune évaluation formelle, ils sont peu examinés tant par les CE que par les AC.

Ce même constat est partagé par les investigateurs qui reçoivent pour tous les essais conduits sur le site un nombre impressionnant de cas graves individuels, et qui n'ont pas le temps de prendre connaissance de toutes ces informations.

Au total beaucoup de données de vigilance circulent, mais leur format et leur périodicité varient selon les États-membres et semblent souvent peu adaptés au regard des missions et des attentes des parties concernées. On peut par conséquent s'interroger sur l'intérêt de la fourniture de telles données quant à la protection des participants à la recherche, d'autant que les CE n'ont en général ni l'expertise, ni les ressources, ni l'organisation pour gérer cette masse d'informations.

\section{1. Les recommandations des promoteurs industriels}

Un premier niveau d'améliorations pourrait être proposé sans avoir à modifier le texte de la Directive :

- Il s'agirait d'harmoniser davantage l'application de la Directive en Europe par l'élaboration de définitions communes et de recommandations explicatives complémentaires : définition du médicament expérimental, des modifications substantielles et du type de recherche (observationnelle, interventionnelle).

- Des recommandations seraient également souhaitables sur le contenu de l'information et du consentement patient, ainsi que sur le remplissage du formulaire de demande EudraCT. Ces définitions et recommandations pourraient être élaborées et validées en commun par les représentants des États-membres au niveau du Clinical Trial Facilitating Group (CTFG) afin qu'elles soient acceptées uniformément dans tous les pays.

- Il conviendrait de clairement définir le contenu du dossier soumis aux $\mathrm{AC}$ et celui adressé aux $\mathrm{CE}$ afin qu'ils ne fassent pas l'objet de demandes nationales supplémentaires et qu'ils soient suffisamment précis pour éviter des interprétations locales divergentes.

- Une attribution claire des responsabilités respectives des deux autorités (AC et $\mathrm{CE}$ ) en termes d'évaluation permettrait d'éviter les demandes contradictoires. Enfin, un guichet unique européen commun pour le dépôt du dossier de demande serait 
fortement souhaitable et l'utilisation de la voie électronique devrait être développée pour mieux communiquer avec les CE.

- À court terme, il serait déjà très utile d'appliquer cette harmonisation et cette simplification au niveau français. Deux propositions discutées lors de la table ronde pourraient rapidement faire l'objet d'un consensus. Il s'agirait d'une part d'un « Avis type du CPP pour une étude sur le médicament » qui permettrait de retrouver dans l'avis rendu par tous les CPP toutes les informations obligatoires et décrites dans l'arrêté du 24 Mai 2006 modifié. Par ailleurs les demandes différentes émanant des CPP ne devraient pas être acceptables car un arrêté (opposable) fixe en France le contenu du dossier CPP pour une étude clinique sur le médicament. Des modalités de dépôt communes devraient être proposées (nombre d'exemplaires, calendrier de soumission).

- Pour la pharmacovigilance, il serait utile de simplifier les obligations en réduisant les déclarations non nécessaires et en évitant de dupliquer les activités relevant des AC. Il faudrait aussi que les modalités de déclaration des effets indésirables graves inattendus soient identiques au regard de la définition dans tous les États-membres.

Un deuxième niveau d'améliorations nécessiterait des modifications des textes au niveau européen (modification de la Directive) :

- Des changements significatifs pourraient être proposés comme une autorisation communautaire de recherche biomédicale sur le modèle des procédures européennes d'enregistrement (procédure centralisée ou décentralisée). Cette nouvelle procédure serait une alternative au processus national actuellement en vigueur. En effet, le type de procédure doit rester in fine au choix du promoteur. Cette alternative permettrait de répondre à plusieurs écueils aujourd'hui rencontrés par les promoteurs en termes de temps, de lourdeur administrative et de surcoût.

- Des modifications significatives pourraient être apportées en pharmacovigilance, afin de maintenir l'accès des CE à l'ensemble des données individuelles de vigilance, sans les surcharger d'informations systématiques inutiles. Il est suggéré de donner l'accès des CE à Eudravigilance (CT module) et de supprimer ainsi l'envoi régulier des suspicions d'effets indésirables graves inattendus ainsi que les listes semestrielles. Il est important de rappeler que seules les AC ont un pouvoir de police sanitaire au vu des données de sécurité. En ce qui concerne le Rapport Annuel de Sécurité qui est en cours d'évolution au profit du Developpement Safety Update Report (DSUR), seul le résumé de ce rapport serait transmis une fois par an aux CE.

\section{Proposition de nouvelle approche basée sur le risque ajouté par la recherche}

Il apparaît nécessaire aujourd'hui d'essayer de définir une nouvelle approche basée sur le risque ajouté par la recherche par rapport aux pratiques de soins courants. Même si le sujet a une portée européenne, et que certains CE de pays comme l'Autriche ont évolué dans ce sens, nous n'envisagerons pour le moment que le cas des recherches en France.

Il existe en fait 3 types de conséquences possibles d'une approche, éventuellement assouplie, basée sur le risque ajouté par la recherche:

- Conséquences sur la sécurité des patients participant à l'étude.

- Conséquences sur la qualité des données :

- en raison de l'impact sur un dossier de mise sur le marché s'il s'agit d'un médicament (ou d'un dispositif médical),

- en raison de l'impact potentiel des résultats de la recherche, par le biais d'une publication ou d'une conférence de consensus, sur la modification de la prise en charge (diagnostique et/ou thérapeutique) d'une pathologie donnée.

- Conséquences médiatiques, d'image de marque ou financières.

Seules les 2 premières approches (sécurité des participants, qualité des données) ont été retenues par la table ronde.

Plusieurs grilles du risque ajouté par la recherche pour la sécurité des patients ont déjà été proposées notamment en France :

- Une première grille à 3 niveaux a été élaborée lors des ateliers de Giens en 2005 en vue d'une adaptation du monitoring. ${ }^{[7]}$

- Une grille à 4 niveaux a été développée par l'Assistance Publique-Hôpitaux de Paris (AP-HP) et mise en place pour adapter le monitoring au risque ajouté par la recherche pour les patients. Cette grille a été acceptée par l'Agence française de sécurité sanitaire des produits de santé (Afssaps) et est utilisée par d'autres promoteurs.

- le risque A correspond au risque le plus faible (en pratique prise de sang, ou examen non invasif),

- le risque D correspond au risque le plus élevé (essais de nouveaux médicaments ou dispositifs médicaux, thérapie génique, thérapie cellulaire). Il est associé à un monitoring de $100 \%$ des dossiers (sur les données importantes),

- les risques $\mathrm{B}$ et $\mathrm{C}$ sont intermédiaires.

Pour un essai de médicament le niveau de risque habituel est C ou D et il ne peut être B que s'il s'agit d'un essai évaluant un médicament ayant déjà l'Autorisation de Mise sur le Marché (AMM) et utilisé dans son indication. Le niveau de risque influe aussi directement sur la décision ou non de mettre en place un Comité de surveillance indépendant (DSMB) qui est systématique en 
Tableau II. Liste non exhaustive des processus pouvant être adaptés selon une approche basée sur le risque ajouté par la recherche.

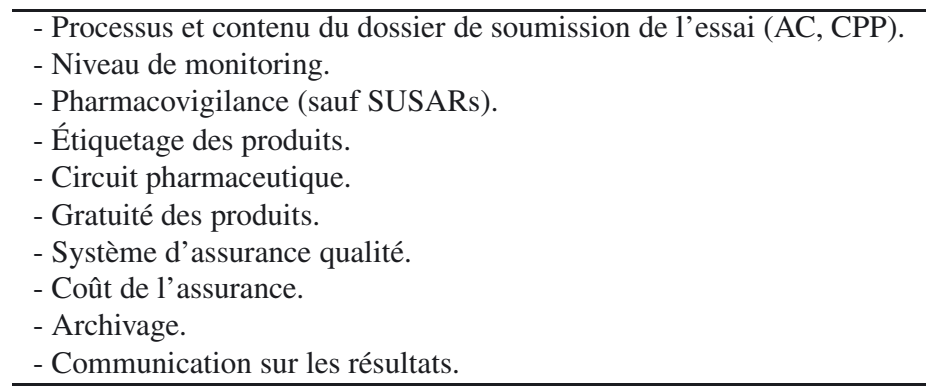

AC : Autorités Compétentes ; CPP : Comités de Protection des Personnes; SUSARs : suspicions d'effets indésirables graves inattendus.

cas de risque $\mathrm{D}$, à discuter en cas de risque $\mathrm{C}$ et non nécessaire en cas de risque $\mathrm{A}$ ou $\mathrm{B}$. Des dérogations à la fourniture gratuite des médicaments par les promoteurs institutionnels sont prévues par l'article L 1121-16-1 du Code de la Santé Publique (CSP) et notamment dans le cas où l'essai concerne une ou plusieurs classes pharmacologiques dont le choix est laissé à l'appréciation de l'investigateur mais restant dans le cadre d'une indication en accord avec l'AMM. Des méthodes de traçabilité des traitements à l'essai peuvent être acceptées par l'Afssaps.

- Une simplification de la grille de l'AP-HP en 3 niveaux a été élaborée en 2009 par un groupe de travail AP-HP et INSERM et elle est en cours de discussion au sein de la coordination des promoteurs institutionnels (CPI).

Bien qu'il n'existe pas d'instrument a priori pour déterminer le niveau de risque avant l'analyse complète de la documentation par l'AC ou le CPP, certains essais pourraient faire l'objet d'une autoévaluation par le promoteur de ce niveau de risque en fonction de critères à définir. Le promoteur adapterait ainsi son monitoring et ses ressources au niveau de risque escompté selon les niveaux retenus pour chaque projet de recherche biomédicale :

1. Selon le niveau de risque ajouté par la recherche pour les patients, qui est celui déclaré aux autorités et à l'assureur. Le monitoring minimal proposé doit s'assurer du respect de la protection et des droits du patient ainsi que de sa sécurité : information et consentement, déviations au protocole, dispensation du traitement éventuel, effets indésirables graves. Ce monitoring doit permettre d'amender éventuellement le protocole afin de mieux protéger le patient (modifications des critères d'éligibilité, du suivi, de la posologie du traitement éventuel) et permettre aussi d'apporter des informations à un éventuel Comité de surveillance indépendant chargé ensuite de faire des recommandations (voire de demander l'arrêt prématuré de l'essai).

2. Selon le niveau de contrôle de qualité des données souhaité (que l'essai soit risqué ou non pour le patient) en fonction de certaines variables (essai pour un dossier d'enregistrement où les données sont rachetées par un industriel; impact important sur la prise en charge médicale). En pratique, il s'agit de savoir si on veut vérifier ou non la qualité des données importantes pour $100 \%$ des patients.

En pratique, on peut donc avoir un risque pour les patients jugé «minime» déclaré aux autorités, mais faire un monitoring à $100 \%$ parce que les données vont êtres cédées à un industriel ou parce qu'elles peuvent avoir un impact international ou modifier la prise en charge des patients (si l'on veut être certain de la véracité et de la fiabilité de ces données et des conclusions).

Pour les études sur le médicament disposant d'une AMM dans l'indication (par exemple les essais de type «stratégie thérapeutique médicament»), les promoteurs institutionnels pourraient simplifier une partie de l'organisation.

Dans tous les cas les conditions suivantes doivent être respectées :

- Il faut garantir le respect des BPC quelque soit le niveau de monitoring.

- Les responsabilités du promoteur doivent rester les mêmes (consentement, déclaration des effets indésirables graves).

En outre, il reste à savoir qui doit valider le niveau de risque proposé par le promoteur (cela ne devrait-il pas être l'AC plutôt que le CE ?) et quelles sont les conditions d'assurance et de réparation (le coût de l'assurance peut en effet varier selon le niveau de risque annoncé pour chaque étude).

Le sujet est d'actualité et mériterait que toutes les expériences européennes en France (projet Optimon), ${ }^{[8]}$ en Angleterre (approche du risque en fonction de l'essai proposée dans la boîte à outil du MRC), ${ }^{[9]}$ en Autriche (CE de Vienne) ${ }^{[10]}$ ou en Allemagne (projet Adamon) ${ }^{[11]}$ soient analysées.

La Table Ronde a listé les points qui pourraient être adaptés dans cette approche basée sur le risque (tableau II). 
Tableau III. Principales recommandations de la table ronde.

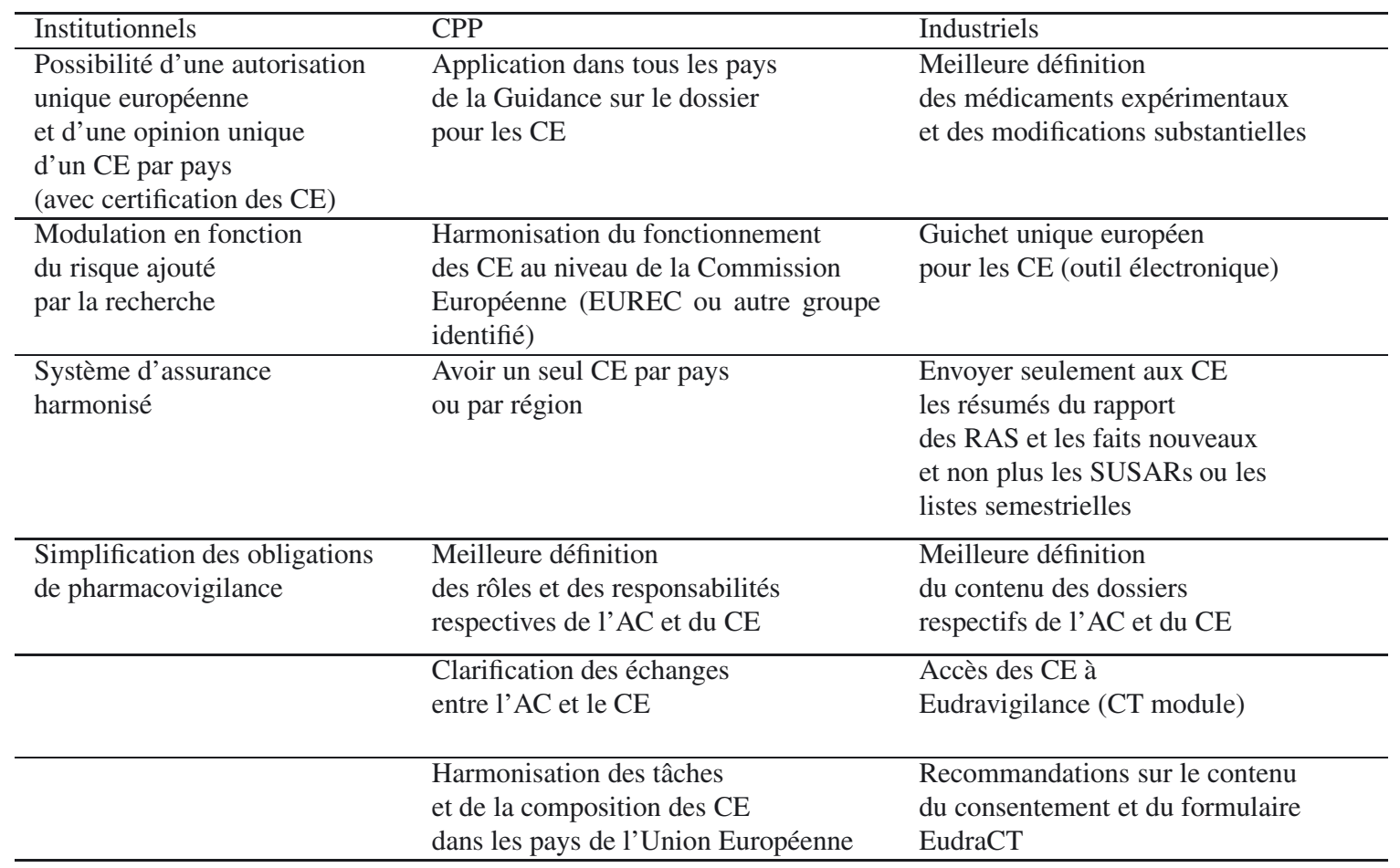

AC : Autorités Compétentes; CE : Comités d'Éthique; CPP : Comité de Protection des Personnes ; EudraCT : base de données des États membres ; EUREC : European Network of Research Ethics Committees; RAS : Rapport Annuel de Sécurité ; SUSARs : suspicions d'effets indésirables graves inattendus

\section{Synthèse des recommandations}

Il importe de rappeler les nombreux acquis de la Directive qu'il convient de conserver : les délais d'autorisation, la possibilité de procédures nationales d'autorisation d'essai, la pharmacovigilance et enfin les principes de protection des personnes et des règles de $\mathrm{BPC}$.

Les principales recommandations faites par la table ronde, sans préjuger des modalités de prise en compte (modifications des recommandations ou du texte de la Directive), sont reprises dans le tableau III.

En pratique, en plus de l'approche basée sur le risque détaillée plus haut, des recommandations à deux niveaux ont été proposées :

\section{1. Améliorer l'application de la Directive}

Un grand nombre de ces propositions pourrait être mise en application sans modifier la Directive mais seulement certaines guidances (par exemple la définition des médicaments expérimentaux ou des modifications substantielles ou l'amélioration du fonctionnement des CE et de l'harmonisation entre les CE). Il serait en revanche impératif de faire appliquer la Directive et ses textes dérivés dans tous les pays et la Commission Européenne devrait en vérifier la mise en œuvre. Enfin pour ce qui concerne les CE, il faudrait clarifier et renforcer le rôle possible de coordination par l'EUREC comme le fait le CTFG pour les AC.

\subsection{Proposer des modifications de la Directive}

Plusieurs points de la Directive nécessiteraient sans doute des changements significatifs donc, soit une révision de celle-ci, soit l'adoption d'un Règlement Européen.

C'est le cas notamment pour la définition des rôles respectifs des $\mathrm{CE}$ et des $\mathrm{AC}$ ou pour la nature de l'information de pharmacovigilance à envoyer aux $\mathrm{CE}$. Ce dernier point a fait l'unanimité des membres de la table ronde. Il s'agirait de donner l'accès aux CE à Eudravigilance (CT module) qui est la base de données européenne sur les effets indésirables survenant au cours de l'essai, ce qui permettrait de diminuer la documentation que les $\mathrm{CE}$ auraient à recevoir. L'envoi systématique de tous les SUSARs et des listes semestrielles serait supprimé et les CE recevraient un 
résumé du RAS. Cette simplification documentaire serait souhaitable non seulement en raison des ressources limitées des CE mais aussi parce que ceux-ci n'ont pas de pouvoir de police sanitaire contrairement aux AC.

En vue d'une meilleure harmonisation, il serait utile aussi d'envisager la possibilité d'une autorisation communautaire de l'essai (autorisation centralisée et/ou décentralisée) sur le modèle des procédures d'AMM, mais tout en gardant au choix du promoteur l'option de déposer une demande d'autorisation d'un essai au niveau national.

Les expériences actuelles de partage des tâches sur les rapports périodiques actualisés de pharmacovigilance (PSUR) ou les travaux du CTFG et notamment la mise en place de la Procédure Volontaire d'Harmonisation (VHP) sont des initiatives qui montrent la prise de conscience des États-membres et leur volonté de concevoir une approche d'évaluation commune partagée.

Une procédure centralisée entraînerait toutefois moins de flexibilité, des délais plus figés, des lourdeurs potentielles méconnues et la disparition des liens nationaux entre AC et CE. Elle entraînerait les mêmes difficultés pour chaque amendement substantiel. Enfin, la mise en place d'une procédure d'autorisation communautaire entraînerait de facto pour la vigilance une évaluation centralisée par une ou deux AC, comme cela est pratiqué dans le cadre de l'évaluation des PSUR. Cette évaluation commune pourrait s'appliquer aux rapports annuels, mais aussi aux faits nouveaux de sécurité voire à l'ensemble des suspicions d'effets indésirables graves inattendus ; dans ce dernier cas cela nécessiterait obligatoirement un document de référence identique dans tous les pays concernés par l'essai et une définition du médicament expérimental, applicable de manière identique dans tous les États-membres.

Les changements proposés pourraient être réalisés dans le cadre d'un Règlement Européen. L'avantage serait, une fois un compromis obtenu au plan européen et le texte voté, d'éviter la transposition ultérieure dans la réglementation de chaque pays, transposition source de dysharmonies potentielles.

Il faut cependant reconnaître les difficultés d'ordre légal, réglementaire, juridique qui en découleraient et qu'il conviendrait de résoudre au préalable. Une approche des représentants parlementaires ou des structures européennes comme le CTFG ou l'EUREC serait nécessaire à la levée de ces difficultés.

\section{Conclusion}

Les travaux de la table ronde ont permis de dégager des constats assez proches des divers partenaires de la recherche sur les avancées ainsi que sur les améliorations ou changements souhaitables de la Directive Européenne 2001/20/CE. Au-delà de l'harmonisation nécessaire entre les pays, la possibilité d'une autorisation communautaire nécessiterait un règlement communautaire. Pour aller plus loin, des contacts seront certainement nécessaires avec des représentants français au parlement européen ou avec les structures de coordination communautaire (comme le CTFG ou le IWGGCP pour les AC, l'EUREC pour les CE, l'ECRIN pour représenter les institutionnels et l'EFPIA pour les industriels). Les discussions seront enrichies en 2010 par les réponses faites par ces organismes à la récente consultation publique sur la Directive. ${ }^{[12]}$

Il serait utile, dans le contexte des réflexions sur l'attractivité de la recherche clinique, de mettre au point des indicateurs de performance au niveau national et européen pour contribuer à l'évaluation des modifications règlementaires qui auront été décidées sur les recherches biomédicales.

Enfin la réflexion sur l'approche du risque adaptée à chaque type d'essai qui fait déjà débat au niveau académique a été largement abordée pendant la table ronde en essayant de s'attacher aux divers points de vue académique, règlementaire mais aussi industriel.

Participants. Michel Abiteboul (Laboratoire Quintiles), Delphine Bertram (Délégation à la Recherche Clinique, CHU, Lyon), Catherine Cornu (Ecrin, CIC, CHU, Lyon), Olivier Chassany (DRRC, AP-HP, Hôpital Saint-Louis Paris), Jacques Demotes-Mainard (Inserm, Paris), Jean-Paul Demarez (Laboratoire Pierre Fabre Médicaments), Vincent Diebolt (GipCengeps, Lyon), Claude Dubray (CIC, CHU, Clermont-Ferrand), Mats Ericson (Laboratoire Amgen), Elisabeth Frija Orvoen (Pathologies du sommeil, Hôpital Pitié-Salpêtrière, Paris), François Hirsch (Inserm, Paris), Yves Juillet (Leem, Paris), Véronique Lamarque (Laboratoire Pfizer), Rémi Le Coent (Laboratoire GlaxoSmithKline), Christian Libersa (CIC, Inserm, Hôpital Cardiologique, Lille), Christine Marey (Science Union, les Laboratoires Servier), Brigitte Marchenay (Laboratoire Roche), Armelle Mijonnet (Laboratoire Merck Sharpe \& Dohme Chibret), Valérie Plattner (Direction Recherche Clinique et Innovation, CHU, Lyon), Anne Raison (Afssaps, Saint-Denis), Patrick Rossignol (CIC, Inserm, CHU, Vandœuvre-lès-Nancy), Philippe Rusch (Faculté de Médecine, Saint-Étienne), Claire Sibenaler (Leem), Tabassome Simon (CHU Saint-Antoine, URC Est Pharmacologie, Paris).

\section{Références}

1. Pehboeck D, Hohlrieder M, Wenzel V, et al. Submission of clinical studies to Ethics Committees or clinical trials registers - the authors' point of view. Intensive Care Med 2009; 35: 713-6

2. European Forum for Good Clinical Practice (EFGCP).Conference on the Impact on Clinical Research of European Legislation (FP7 program, ICREL), results § discussion, Brussels 2 Dec 2008. http: //www. efgcp . 
be/Downloads/confDocuments/Programme_ICREL_2_Dec_2008_ final.pdf

3. Kubiak C, De Andres-Trelles F, Kuchinke W, et al. Common definition for categories of clinical research: a prerequisite for a survey on regulatory requirements by the European Clinical Research Infrastructures Network (ECRIN).Trials 2009; 10: 95-101

4. Jardé O. Projet de Loi sur les recherches sur la personne. Assemblée Nationale, 22 janvier 2009. http: //www . assemblee-nationale. fr

5. Clinical Trials Facilitation Group: CTA assessment in member states, 22 December 2008. http://www.hma.eu/uploads/media/Assessment_ in_MS_public_dec_08__2_B.pdf.

6. European Network of Research Ethics Committees (EUREC). http: //www . eurecnet.org/index.html

7. Bertoye PH, Courcier-Duplantier S, Best N. Adaptation de la mise en oeuvre des bonnes pratiques cliniques en fonction des caractéristiques de certaines recherches. Thérapie 2006; 61: 271-7

8. Projet Optimon, OPTimisation du MONitorage : évaluation de l'efficacité et du coût de deux stratégies de monitorage pour la recherche clinique institutionnelle. Version du 4 février 2008. https://ssl2.isped. u-bordeaux 2 . fr/optimon/Documents . aspx
9. Notes on good practice for research organisations in the management of a portfolio of trials 2: assessment of risk. Clinical trials tool kit issued by the Department of Health and the Medical Research Council (MRC), 2004. http://www.cttoolkit.ac.uk/_db/_documents/MPTrials2.pdf

10. Wolzt M, Druml C, Leitner D, et al. Protocols in expedited review-tackling the workload of Ethics Committees. Intensive Care Med 2009; 35: 613-5

11. Brosteanu $\mathrm{O}$, Houben $\mathrm{P}$, Ihrig $\mathrm{K}$, et al. Risk analysis and risk adapted on-site monitoring in non commercial clinical trials. Clin Trials 2009; 6: 585-96

12. European Commission. Assessment of the functioning of the Clinical Trials Directive 2001/20/EC: public consultation paper ENTR/F/2/SF D (2009) 32674. 09/10/2009. http://ec.europa.eu/enterprise/sectors/ pharmaceuticals/files/clinicaltrials/docs/2009_10_09_ public-consultation-paper.pdf

Correspondance et offprints : Pascal Bilbault, Recherche Clinique Boehringer Ingelheim France, 12 rue André Huet, 51060 Reims Cedex, France. E-mail : pascal.bilbault@boehringer-ingelheim.com 